\title{
RELAXATION FOR QUASI-LINEAR DIFFERENTIAL INCLUSIONS IN NON SEPARABLE BANACH SPACES
}

\author{
Aurelian Cernea \\ Faculty of Mathematics, University of Bucharest \\ Academiei 14, 70109 Bucharest, Romania \\ acernea@math.math.unibuc.ro
}

\begin{abstract}
We consider a Cauchy problem for nonconvex quasi-linear differential inclusions in non separable Banach spaces and we prove that the set of mild solutions of this problem is dense in the set of mild solutions of the convexified problem.
\end{abstract}

Keywords: quasi-linear inclusions, mild solutions, Lusin measurable multifunctions, relaxation property.

\section{Introduction}

In this paper we consider quasi-linear differential inclusions of the form

$$
x^{\prime}(t) \in A(t, x(t)) x(t)+F(t, x(t)), \quad \text { a.e. }([0, T]), \quad x(0)=a,
$$

where $A(t, w)$ is a linear operator from the real Banach space $X$ in $X$, $t \in I:=[0, T], w$ belongs to an open and nonempty set $D \subset X$ and $F$ is a set-valued map from $I \times X$ to $\mathcal{P}(X)$.

Qualitative properties and structure of the set of solutions of this problem have been studied by many authors ([1], [2], [8], [9], [10], [12] etc). In [9] it is shown that if $X$ is a separable Banach space, the set of mild solutions of the problem (1.1) is dense in the set of mild solutions of the convexified (relaxed) problem:

$$
x^{\prime}(t) \in A(t, x(t)) x(t)+\overline{c o} F(t, x(t)), \quad \text { a.e. }([0, T]), \quad x(0)=a .
$$

If the operator $A$ depends neither on $t$ nor on $w$ the quasi-linear inclusions (1.1)-(1.2) reduce to the corresponding semilinear problems

$$
x^{\prime}(t) \in A x(t)+F(t, x(t)), \quad \text { a.e. }([0, T]), \quad x(0)=a,
$$

The original version of this chapter was revised: The copyright line was incorrect. This has been corrected. The Erratum to this chapter is available at DOI: 10.1007/978-0-387-35690-7_44 


$$
x^{\prime}(t) \in A x(t)+\overline{c o} F(t, x(t)), \quad \text { a.e. }([0, T]), \quad x(0)=a .
$$

Recently, De Blasi and Pianigiani ([4]) established a relaxation result for the problems (1.3), (1.4) in an arbitrary, not necessarily separable, Banach space $X$. Even if the general ideas of proving a relaxation theorem are still present, the approach in [4] has a fundamental difference which consists in the construction of the measurable selections of the multifunction. This construction does not use classical selection theorems as Kuratowsky and Ryll-Nardzewski ([7]) or Bressan and Colombo ([3]).

The aim of this paper is to extend the result in [4] to the more general problem (1.1). We will prove the relaxation property of mild solutions for problem (1.1) in an arbitrary space $X$. The proof of our main result follows the general ideas in [4] and [9].

\section{Preliminaries}

Consider $X$ an arbitrary real Banach space with norm $\|$.$\| and let$ $\mathcal{P}(X)$ be the space of all bounded nonempty subsets of $X$ endowed with the Hausdorff pseudometric

$$
d_{H}(A, B)=\max \left\{d^{*}(A, B), d^{*}(B, A)\right\}, \quad d^{*}(A, B)=\sup _{a \in A} d(a, B),
$$

where $d(x, A)=\inf _{a \in A}\|x-a\|, A \subset X, x \in X$.

Let $\mathcal{L}$ be the $\sigma$-algebra of the (Lebesque) measurable subsets of $R$ and, for $A \in \mathcal{L}$, let $\mu(A)$ be the Lebesque measure of $A$.

Let $Y$ be a metric space. An open (resp. closed) ball in $Y$ with center $y$ and radius $r$ is denoted by $B_{Y}(y, r)$ (resp. $\bar{B}_{Y}(y, r)$ ). For any set $A \subset Y$ we denote by $\overline{c o} A$ the closed convex hull of $A$. In what follows $B=B_{X}(0,1)$ and $I=[0,1]$.

A multifunction $F: Y \rightarrow \mathcal{P}(X)$ with closed bounded nonempty values is said to be $d_{H}$-continuous at $y_{0} \in Y$ if for every $\epsilon>0$ there exists $\delta>0$ such that for any $y \in B_{Y}\left(y_{0}, r\right)$ we have $d_{H}\left(F(y), F\left(y_{0}\right)\right) \leq \epsilon$. $F$ is called $d_{H}$-continuous if it is so at each point $y_{0} \in Y$.

Let $A \in \mathcal{L}$, with $\mu(A)<\infty$. A multifunction $F: A \rightarrow \mathcal{P}(X)$ with closed bounded nonempty values is said to be Lusin measurable if for every $\epsilon>0$ there exists a compact set $K_{\epsilon} \subset A$, with $\mu\left(A \backslash K_{\epsilon}\right)<\epsilon$ such that $F$ restricted to $K_{\epsilon}$ is $d_{H}$-continuous.

It is clear that if $F, G: A \rightarrow \mathcal{P}(X)$ and $f: A \rightarrow X$ are Lusin measurable then so are $F$ restricted to $B$ ( $B \subset A$ measurable), $F+G$ and $t \rightarrow d(f(t), F(t))$. Moreover, the uniform limit of a sequence of Lusin measurable multifunctions is also Lusin measurable.

As usual we denote by $L^{1}(I, X)$ the Banach space of all Bochner integrable functions $x():. I \rightarrow X$ endowed with the norm $\|x(.)\|_{1}=$ $\int_{0}^{1}\|x(s)\| d s$. In what follows we shall use the following assumptions. 
Hypothesis 2.1. a) $F: I \times X \rightarrow \mathcal{P}(X)$ is a set-valued map with closed bounded nonempty values and for any $x \in X, F(., x)$ is Lusin measurable on $I$.

b) There exists an integrable function $k(.) \in L^{1}(I, R)$ such that

$$
d_{H}(F(t, x), F(t, y)) \leq k(t)\|x-y\| \quad \forall(t, x),(t, y) \in I \times X .
$$

c) There exists $q(.) \in L^{1}(I, R)$ such that for any continuous function $x(.) \in C(I, D)$ and any $t \in I$ we have

$$
F(t, x(t)) \subset q(t) B .
$$

A family of bounded linear operators $\mathcal{U}(t, s)$ on $X, 0 \leq s \leq t \leq 1$ depending on two parameters is said to be an evolution system ([11]) if the following conditions are satisfied

1) $\mathcal{U}(s, s)=1, \mathcal{U}(t, r) \mathcal{U}(r, s)=\mathcal{U}(t, s) 0 \leq s \leq r \leq t \leq T$

2) $(t, s) \rightarrow \mathcal{U}(t, s)$ is strongly continuous, i.e.

$$
\lim _{t \searrow s} \mathcal{U}(t, s) x=x, \quad \forall x \in X
$$

Hypothesis 2.2. a) $X$ is a real Banach space and $D \subset X$ is an open nonempty set.

b) For any $u \in C(I, D)$ the family of linear operators $\{A(t, u), t \in I\}$ generates a unique strongly continuous evolution system $\mathcal{U}_{u}(t, s)$, $0 \leq s \leq t \leq T$.

c) If $u \in C(I, D)$, the evolution system $\mathcal{U}_{u}(t, s), 0 \leq s \leq t \leq T$ satisfies:

there exists $M \geq 0$ such that $\left\|\mathcal{U}_{u}(t, s)\right\| \leq M, 0 \leq s \leq t \leq T$, uniformly in $u$;

for any $u, v \in C(I, D)$ and any $w \in D$ we have

$$
\left\|\mathcal{U}_{u}(t, s) w-\mathcal{U}_{v}(t, s) w\right\| \leq M\|w\| \int_{s}^{t}\|u(\tau)-v(\tau)\| d \tau
$$

By a mild solution of the Cauchy problem (1.1) we mean a function $x():. I \rightarrow X$ satisfying the following conditons:

i) $x($.$) is continuous on I$ with $x(0)=a$

ii) there exists a Lusin measurable function $f():. I \rightarrow X$, Bochner integrable such that

$$
\begin{gathered}
f(t) \in F(t, x(t)), \quad \forall t \in I, \\
x(t)=\mathcal{U}_{x}(t, 0) a+\int_{0}^{t} \mathcal{U}_{x}(t, s) f(s) d s, \quad \forall t \in I .
\end{gathered}
$$


According to [4] in the above definition the Lusin measurability of $f($.$) is equivalent to the (strong) measurability of f($.$) .$

We recall some results we shall use in the sequel.

Lemma 2.3. ([4]) Let $F: I \times X \rightarrow \mathcal{P}(X)$ be a set-valued map with closed bounded nonempty values that satisfies Hypothesis 2.1. Then, for any $x():. I \rightarrow X$ continuous, $u():. I \rightarrow X$ measurable and $\epsilon>0$ we have:

a) the multifunction $t \rightarrow F(t, x(t))$ is Lusin measurable on $I$.

b) the multifunction $G: I \rightarrow \mathcal{P}(X)$ defined by

$$
G(t):=(F(t, x(t))+\epsilon B) \cap B_{X}(u(t), d(u(t), F(t, x(t)))+\epsilon)
$$

has a Lusin measurable selection $f: I \rightarrow X$.

Lemma 2.4. ([9]) Suppose that Hypothesis 2.2 is satisfied and that each quasi-linear Cauchy problem

$$
x_{n}^{\prime}(t) \in A\left(t, x_{n}(t)\right) x_{n}(t)+f_{n}(t), \quad \text { a.e. }([0, T]), \quad x_{n}(0)=a,
$$

$n \in N$, has a mild solution

$$
x_{n}(t)=\mathcal{U}_{x_{n}}(t, 0) a+\int_{0}^{t} \mathcal{U}_{x_{n}}(t, s) f_{n}(s) d s, \quad t \in I .
$$

Suppose, also, that there exists $x \in C(I, X)$ and $f \in L^{1}(I, X)$ such that $x_{n} \rightarrow x$ in $C(I, X), f_{n} \rightarrow f$ in $L^{1}(I, X)$ and the set $\{f\} \cup\left\{f_{n}\right\}_{n \in N}$ is integrably bounded by a function $m \in L^{1}(I, X)$. Then,

$$
x(t)=\mathcal{U}_{x}(t, 0) a+\int_{0}^{t} \mathcal{U}_{x}(t, s) f(s) d s, \quad \forall t \in I .
$$

\section{The main result}

In order to prove our main result we need the following lemma which is a quasi-linear version of Lemma 4.2 in [4], obtained for linear differential inclusions. The proof can be easily performed through the same arguments employed to establish Lemma 4.2 in [4].

Lemma 3.1. We assume that Hypotheses 2.1-2.2 are satisfied. Let $a \in X$ and let $y():. I \rightarrow X$ be a mild solution of the relaxed problem (1.2). Then, for any $0<\sigma<1$ there exists a mild solution $x_{0}():. I \rightarrow X$ of the Cauchy problem

$$
x^{\prime}(t) \in A(t, x(t)) x(t)+F(t, x(t))+\phi_{\sigma}(t) B, \text { a.e. }([0, T]), x(0)=a,
$$

where $\phi_{\sigma}(.) \in L^{1}(I,[0, \infty))$ with $\int_{0}^{1} \phi_{\sigma}(t) d t<2 \sigma$, such that

$$
\left\|x_{0}(t)-y(t)\right\|<\sigma, \quad \forall t \in I .
$$


Our main result states that the set of mild solutions of the problem (1.1) is dense in the set of mild solutions of the convexified (relaxed) problem (1.2).

Theorem 3.1. We assume that Hypotheses 2.1-2.2 are satisfied. Let $a \in X$ and let $y():. I \rightarrow X$ be a mild solution of the convexified problem (1.2). Then, for every $\epsilon>0$ there exists a mild solution $x():. I \rightarrow X$ of the problem (1.1) such that:

$$
\|x(t)-y(t)\|<\epsilon, \quad \forall t \in I .
$$

Proof. Let $y():. I \rightarrow X$ be an arbitrary mild solution of the Cauchy problem (1.2) and let $0<\epsilon<1$. We define

$$
L(t):=\int_{0}^{t} M\left(\|a\|+\|q\|_{1}+\epsilon+k(s)\right) d s, \quad t \in I .
$$

Fix $\sigma$ such that $0<\sigma<\frac{\epsilon}{(3 M+1) e^{L(1)}}$. Let $\phi_{\sigma}(.) \in L^{1}(I,[0, \infty))$ such that $\int_{0}^{1} \phi_{\sigma}(t) d t<2 \sigma$.

By Lemma 3.1 there exists a mild solution $x_{0}():. I \rightarrow X$ of the problem (3.1) such that:

$$
\left\|x_{0}(t)-y(t)\right\|<\sigma, \forall t \in I .
$$

By definition of mild solution $x_{0}($.$) is continuous, x_{0}(0)=a$ and there exists a Lusin measurable function $f_{0}():. I \rightarrow X$, Bochner integrable such that

$$
\begin{gathered}
f_{0}(t) \in F\left(t, x_{0}(t)\right)+\phi_{\sigma}(t) B, \quad t \in I, \\
x_{0}(t)=\mathcal{U}_{x_{0}}(t, 0) a+\int_{0}^{t} \mathcal{U}_{x_{0}}(t, s) f_{0}(s) d s, \quad t \in I .
\end{gathered}
$$

Let $\sigma_{n}=\frac{\sigma}{2^{n+2}}$ and $p_{0}(t):=d\left(f_{0}(t), F\left(t, x_{0}(t)\right)\right), t \in I$.

Since $x_{0}($.$) is continuous, by Lemma 2.3$ there exists a Lusin measurable function $f_{1}():. I \rightarrow X$ satisfying, for $t \in I$,

$$
f_{1}(t) \in\left(F\left(t, x_{0}(t)\right)+\epsilon_{1} B\right) \cap B_{X}\left(f_{0}(t), d\left(f_{0}(t), F\left(t, x_{0}(t)\right)\right)+\sigma_{1}\right)
$$

Hence $f_{1}($.$) is also Bochner integrable on I$. Define $x_{1}():. I \rightarrow X$ by

$$
x_{1}(t)=\mathcal{U}_{x_{0}}(t, 0) a+\int_{0}^{t} \mathcal{U}_{x_{0}}(t, s) f_{1}(s) d s, \quad \forall t \in I .
$$

By reccurence, we construct a sequence $\left\{x_{n}\right\}_{n}$ of continuous functions $x_{n}: I \rightarrow X, n \geq 2$ given by

$$
x_{n}(t)=\mathcal{U}_{x_{n-1}}(t, 0) a+\int_{0}^{t} \mathcal{U}_{x_{n-1}}(t, s) f_{n}(s) d s, \quad t \in I,
$$


with $f_{n}():. I \rightarrow X$ a Lusin measurable function satisfying, for $t \in I$,

$$
f_{n}(t) \in\left(F\left(t, x_{n-1}(t)\right)+\sigma_{n} B\right) \cap B_{X}\left(f_{n-1}(t), d\left(f_{n-1}(t), F\left(t, x_{n-1}(t)\right)\right)+\sigma_{n}\right) .
$$

From (3.6), for $n \geq 2$, we obtain

$$
\begin{gathered}
\left\|f_{n}(t)-f_{n-1}(t)\right\| \leq d\left(f_{n-1}(t), F\left(t, x_{n-1}(t)\right)\right)+\sigma_{n} \leq \\
\leq d\left(f_{n-1}(t), F\left(t, x_{n-2}(t)\right)\right)+d_{H}\left(F\left(t, x_{n-2}(t)\right), F\left(t, x_{n-1}(t)\right)\right)+\sigma_{n} \\
\leq \sigma_{n-1}+k(t)\left\|x_{n-1}(t)-x_{n-2}(t)\right\|+\sigma_{n} .
\end{gathered}
$$

Since $\sigma_{n-1}+\sigma_{n}<\sigma_{n-2}$ we deduce, for $n \geq 2$, that

$$
\left\|f_{n}(t)-f_{n-1}(t)\right\| \leq \sigma_{n-2}+k(t)\left\|x_{n-1}(t)-x_{n-2}(t)\right\| .
$$

Define $r(t):=M \int_{0}^{t}\left(p_{0}(s)+\sigma\right) d s, \quad t \in I$.

One has

$$
\begin{aligned}
\left\|x_{1}(t)-x_{0}(t)\right\| & \leq \int_{0}^{t}\left\|\mathcal{U}_{x_{0}}(t, s) f_{1}(s)-\mathcal{U}_{x_{0}}(t, s) f_{0}(s)\right\| d s \leq \\
& \leq M \int_{0}^{t}\left(p_{0}(s)+\sigma\right) d s=r(t)
\end{aligned}
$$

Clearly, by (3.3) $p_{0}(t) \leq \phi_{\sigma}(t), \forall t \in I$, hence $\int_{0}^{1} p_{0}(t) d t \leq \int_{0}^{1} \phi_{\sigma}(t) d t<2 \sigma$. Thus, $r(1)=M \int_{0}^{1}\left(p_{0}(s)+\sigma\right) d s<3 M \sigma$.

By reccurence, we shall prove that, for $n \geq 1$, one has

$$
\left\|x_{n}(t)-x_{n-1}(t)\right\| \leq r(t) \frac{(L(t))^{n-1}}{(n-1) !} \quad \forall t \in I .
$$

For $n=1$ the inequality is already proved.

Assuming that (3.8) is valid for $n$, we show that (3.8) holds for $n+1$. Using (3.5)-(3.8) one has

$$
\begin{gathered}
\left\|x_{n+1}(t)-x_{n}(t)\right\| \leq\left\|\mathcal{U}_{x_{n}}(t, 0) a-\mathcal{U}_{x_{n-1}}(t, 0) a\right\|+ \\
\int_{0}^{t}\left\|\mathcal{U}_{x_{n}}(t, s) f_{n+1}(s)-\mathcal{U}_{x_{n-1}}(t, s) f_{n}(s)\right\| d s \leq \\
\leq M\|a\| \int_{0}^{t}\left\|x_{n}(s)-x_{n-1}(s)\right\| d s+\int_{0}^{t}\left\|\mathcal{U}_{x_{n}}(t, s) f_{n+1}(s)-\mathcal{U}_{x_{n}}(t, s) f_{n}(s)\right\| d s+ \\
+\int_{0}^{t}\left\|\mathcal{U}_{x_{n}}(t, s) f_{n}(s)-\mathcal{U}_{x_{n-1}}(t, s) f_{n}(s)\right\| d s \leq \\
\leq M\|a\| \int_{0}^{t}\left\|x_{n}(s)-x_{n-1}(s)\right\| d s+M \int_{0}^{t}\left\|f_{n+1}(s)-f_{n}(s)\right\| d s+
\end{gathered}
$$




$$
\begin{gathered}
+\int_{0}^{t} M\left\|f_{n}(\tau)\right\|\left(\int_{\tau}^{t}\left\|x_{n}(s)-x_{n-1}(s)\right\| d s\right) d \tau \leq \\
\leq M\|a\| \int_{0}^{t}\left\|x_{n}(s)-x_{n-1}(s)\right\| d s+M \int_{0}^{t}\left(\sigma_{n-1}+k(s)\left\|x_{n}(s)-x_{n-1}(s)\right\|\right) d s+ \\
+\int_{0}^{t} M\left(\|q\|_{1}+\sigma_{n}\right)\left\|x_{n}(s)-x_{n-1}(s)\right\| d s \leq \int_{0}^{t}\left[M\left(\|a\|+\|q\|_{1}+\sigma_{n-2}\right)+\right. \\
+M k(s)]\left\|x_{n}(s)-x_{n-1}(s)\right\| d s \leq \int_{0}^{t} L^{\prime}(s)\left\|x_{n}(s)-x_{n-1}(s)\right\| d s \leq \\
\leq \int_{0}^{t} L^{\prime}(s) r(s) \frac{(L(s))^{n-1}}{(n-1) !} d s<r(t) \frac{(L(t))^{n}}{n !} .
\end{gathered}
$$

From (3.8) we obtain

$$
\begin{gathered}
\left\|x_{n}(t)-x_{0}(t)\right\| \leq \sum_{k \geq 1}\left\|x_{k}(t)-x_{k-1}(t)\right\| \leq \\
\leq r(t) \sum_{k=1}^{n-1} \frac{(L(t))^{k}}{k !}<r(1) e^{L(1)} .
\end{gathered}
$$

Therefore

$$
\left\|x_{n}(t)-x_{0}(t)\right\| \leq 3 M e^{L(1)} \sigma, \quad t \in I .
$$

On the other hand, from (3.4) it follows that

$$
\left\|x_{n+1}(t)-x_{n}(t)\right\| \leq r(1) \frac{(L(1))^{n}}{n !} \quad t \in I .
$$

From (3.10) it follows that the sequence $\left\{x_{n}\right\}_{n}$ converges uniformly on $I$ to a continuous function, $x():. I \rightarrow X$.

In view of of (3.7) we have

$$
\left\|f_{n+1}(t)-f_{n}(t)\right\| \leq \sigma_{n-1}+k(t) r(1) \frac{(L(1))^{n}}{n !}, \quad t \in I,
$$

which implies that the sequence $\left\{f_{n}\right\}_{n}$ converges to a Lusin measurable function $f():. I \rightarrow X$. From (3.6) it follows that

$$
\left\|f_{n}(t)\right\| \leq\|q(t)\|+1 \quad \forall t \in I, n \in N,
$$

hence $f$ is Bochner integrable on $I$.

Letting $n \rightarrow \infty$ and using Lemma 2.4 we conclude that $x($.$) is a mild$ solution of the Cauchy problem (1.1).

From (3.9) we infer that

$$
\left\|x(t)-x_{0}(t)\right\| \leq 3 M e^{L(1)} \sigma, t \in I .
$$


Finally, from the last estimation, (3.2) and the choice of $\sigma$ we deduce

$$
\begin{gathered}
\|x(t)-y(t)\| \leq\left\|x(t)-x_{0}(t)\right\|+\left\|x_{0}(t)-y(t)\right\| \leq \\
\leq(3 M+1) e^{L(1)} \sigma<\epsilon, t \in I
\end{gathered}
$$

and the proof is complete.

Remark 3.3. When the operator $A$ depends neither on $t$ nor on $w$ problem (1.1) reduces to problem (1.3) and Theorem 3.1 yields known results, namely Theorem 4.1 in [4].

\section{References}

[1] Ahmed N. U. and Xiang X.: Optimal control of infinite dimensional uncertain systems, J. Optim. Theory Appl. 80 (1994), 261-272.

[2] Anguraj A. and Balachandran K.: Existence of solutions of nonlinear differential inclusions, Mem. Fac. Sci. Kochi Univ. 13 (1992), 61-66.

[3] Bressan A. and Colombo G.: Extensions and selections of maps with decomposable values, Studia Math. 90 (1988), 69-86.

[4] De Blasi F. S. and Pianigiani G.: Evolution inclusions in non separable Banach spaces, Comment. Math. Univ. Carolinae 40 (1999), 227-250.

[5] Filippov A. F.: Classical solutions of differential equations with multi-valued right hand side, SIAM J. Control 5 (1967), 609-621.

[6] Frankowska H.: A priori estimates for operational differential inclusions, J. Diff. Eqs. 84 (1990), 100-128.

[7] Kuratowski K. and Ryll-Nardzewski C.: A general theorem on selectors, Bull. Acad. Pol. Sci. Math. Astron. Phys. 13 (1965), 397-403.

[8] Muresan M.: On a boundary value problem for quasi-linear differential inclusions of evolutions, Collect Math. 45 (1994), 165-175.

[9] Muresan M.: Qualitative properties of solutions to quasi-linear inclusions II, Pure Math. Appl. 5 (1994), 331-353.

[10] Papageorgiu N. S.: On multivalued evolution equations and differential inclusions in Banach spaces, Comment. Math. Univ. St. Pauli 36 (1986), 21-39.

[11] Pazy A.: Semigroups of linear operators and applications to partial differential equations, Springer, Berlin, 1983.

[12] Sanekata N.: Abstract quasi-linear equations of evolutions in nonreflexive Banach spaces, Hiroshima Math. J. 19 (1989), 109-139. 